\title{
A compact RFQ cooler buncher for CRIS experiments
}

Check for updates

\section{B. S. Cooper ${ }^{1}$ • H. A. Perrett ${ }^{1}$. C. M. Ricketts ${ }^{1}$. C. Read ${ }^{1}$. G. Edwards ${ }^{1} \cdot$ K. T. Flanagan ${ }^{1}$. J. Billowes ${ }^{1}$ - C. L. Binnersley ${ }^{1}$ - M. L. Bissell ${ }^{1}$ - T. E. Cocolios ${ }^{2}$ • R. P. de Groote ${ }^{2}$. G. J. Farooq-Smith ${ }^{2}$ - R. F. Garcia Ruiz ${ }^{3}$ - W. Gins ${ }^{2}$ - A. Koszorus ${ }^{2}$ - G. Neyens Ka $^{2,3}$. F. P-Gustafsson ${ }^{2} \cdot$ H. H. Stroke ${ }^{4}$ A. R. Vernon ${ }^{1} \cdot$ K. D. A. Wendt ${ }^{5} \cdot$ S. G. Wilkins ${ }^{1}$. X. F. Yang ${ }^{2,6}$}

Published online: 13 May 2019

(C) The Author(s) 2019

\begin{abstract}
A compact radio frequency cooler buncher (RFQCB) is currently in development between The University of Manchester, KU Leuven, and CERN. The device will be installed as part of the Collinear Resonance Ionisation Spectroscopy (CRIS) experiment at the Isotope separator On-line device (ISOLDE) at CERN. The purpose of developing a dedicated RFQCB for the CRIS experiment is to increase data collection efficiency, and simplify the process of obtaining reference measurements with stable isotopes. The CRIS technique is outlined in addition to an overview of the proposed RFQCB, and its potential compatibility for implementation at ISOLDE.
\end{abstract}

Keywords Ion trap $\cdot$ Laser spectroscopy $\cdot 3 \mathrm{D}$ printing

This article is part of the Topical Collection on Proceedings of the 7th International Conference on Trapped Charged Particles and Fundamental Physics (TCP 2018), Traverse City, Michigan, USA, 30 September-5 October 2018

Edited by Ryan Ringle, Stefan Schwarz, Alain Lapierre, Oscar Naviliat-Cuncic, Jaideep Singh and Georg Bollen

\section{B. S. Cooper}

ben.cooper@manchester.ac.uk

1 University of Manchester, Oxford Road, Manchester, UK

2 KU Leuven, Instituut voor Kern- en Stralingsfysica, B-3001, Leuven, Belgium

3 EP Department, CERN, CH-1211, Geneva 23, Switzerland

4 Department of Physics, New York University, New York, NY 10003, USA

5 Institut für Physik, Johannes Gutenberg-Universität Mainz, D-55128, Mainz, Germany

6 School of Physics and State Key Laboratory of Nuclear Physics and Technology,

Peking University, Beijing, 100871, China 


\section{Introduction}

The CRIS technique [1] has been shown to be a sensitive method for measuring nuclear observables of exotic isotopes [2-5]. The technique has been used extensively over the last decade to measure nuclear ground and isomeric-state properties including changes in the mean-square charge radii. The method uses a multiple-step laser excitation and ionisation scheme which depends on the element under investigation. Bunched beams are currently delivered to the CRIS experiment from an RFQCB which is part of the ISOLDE facility (ISCOOL) [6, 7]. It provides ion bunches with a narrow energy spread (typically $<1 \mathrm{eV}$ ), temporal bunch widths of $<10 \mu \mathrm{s}$, and transmission efficiencies in bunch mode of up to $70 \%$. ISCOOL has been critical for the success of the CRIS experiment. A dedicated CRIS RFQCB would separate the function of ISOLDE from CRIS and help reduce set up times prior to on-line experiments at the ISOLDE facility. It would enable optimisation of beam transport and beam properties, weeks in advance of radioactive ion beam experiments. The tasks of beam tuning and buncher optimisation has previously taken up to $40 \%$ of on-line studies. It would therefore be extremely beneficial to have these tasks completed in advance. Another advantage of an independent RFQCB would be the ability to perform long studies for the purpose of assessing systematic errors throughout the full experimental setup, which is essential for the study of light elements. Such cases require very precise isotope shift measurements to extract the changes in mean-square charge radii. It would also simplify the process of switching from a radioactive beam to a stable reference isotope since the CRIS ablation ion source [8] could be utilised instead of cycling the magnets of the high resolution separator (HRS). These reference measurements are essential to monitor drifts, assess systematic errors, extract nuclear observables, and must be carried out many times during an experiment. Magnet cycling is currently a time consuming process. It takes over 6 minutes to change mass at the HRS. Depending on the number of reference measurements required which varies between the experiments, up to $10 \%$ of the time spent during an on-line campaign has been spent on magnet cycling.

Magnet cycling and other tasks such as beam tuning and laser alignment, which must be carried out with bunches from ISCOOL currently, limit the data collection time significantly. By removing the dependence on the HRS for reference measurements, and adding the ability to fully optimise the beam bunch quality, and laser overlap ahead of an experiment, the data collection capacity of CRIS will increase considerably. An independent cooler buncher will also enable CRIS to take beam from both the HRS and the general purpose separator (GPS) which currently does not pass though a buncher.

This article outlines the current progress of the project which aims to deliver a fully commissioned and characterised cooler buncher to the CRIS experiment during the second long shutdown at CERN (LS2). During this period the device will be installed and optimised using the CRIS ablation ion source such that it is ready to accept beam from ISOLDE, when the system is returned to a fully operational state in 2021 .

\section{The CRIS experiment at ISOLDE}

At the ISOLDE facility, pulses of $1.4 \mathrm{GeV}$ protons from the proton synchrotron booster (PSB) impinge on a suitable target. At this energy, nuclear reactions take place within the material. The reaction products diffuse into a transfer line which transports them to a 
hot cavity for surface or laser ionisation. Laser ionisation is facilitated by the resonance ionisation laser ion source (RILIS). A low energy ion beam is formed and then mass separated by two dipole magnets that comprise the HRS. The mass of interest is then transported to the cooler-buncher ISCOOL. A cooler-buncher is required for CRIS experiments as the laser system is composed of high power, pulsed lasers which operate at repetition rates between $100 \mathrm{~Hz}$ and $1 \mathrm{kHz}$, with pulse widths between 6 and $50 \mathrm{~ns}$. The ion beam is therefore required to arrive in bunches to remove duty cycle losses. Currently ISCOOL accepts beam from ISOLDE and decelerates it from the target exit energy $(40-60 \mathrm{keV})$ to around $100 \mathrm{eV}$. Helium is used as a buffer gas. The pressure in the trapping region is around 0.1 mbar where the gas kinematically cools the ion beam. Radially the ions are confined within an oscillating RF electric field applied to four rods and a DC drag field is also applied in the standard way for axial confinement. The voltage on a gate electrode is periodically lowered at a time which is suitable for the temporal overlap of the ion beam with laser pulses at a well defined section of the CRIS beamline. Following optimisation of the output pulse from ISCOOL, the ions are transported to a charge exchange cell (CEC) where neutralisation occurs. This requires an alkali vapour, typically of potassium or sodium originating from a heated unit [9]. The neutralisation efficiency is typically around 50\%, and the remaining ions are deflected out of the beam. Typically the atom pulse will undergo a stepwise laser excitation in a differentially pumped interaction region. The pressure in this region is maintained at ultra high vacuum (UHV) $\left(<10^{-9}\right.$ mbar) to reduce background resulting from collisional ionisation. The final step of the laser interaction process is ionisation with a high fluence laser pulse, typically $1064 \mathrm{~nm}$, or $532 \mathrm{~nm}$, or excitation to an autoionising state. Only atoms excited by an initial resonant laser pulse to an excited state can be ionised and detected in this way. Following the interaction region there is a $20^{\circ}$ bend which deflects the final beam to a counting detector. As the excitation is performed collinearly the scheme is essentially Doppler free, and by monitoring the detector count rate as a function of laser frequency, a hyperfine spectrum can be obtained. Analysis of the spectra lead to extraction of the nuclear properties of interest. It is essential that the output of ISCOOL is tuned in such a way that the ion packet has a small emittance, a low energy spread, and a short temporal pulse width. Optimisation of these parameters is critical for ensuring the success of a CRIS measurement as they influence the efficiency of the laser excitation and ionisation schemes as well as the properties of the extracted lineshapes. The optimisation process can take time and once the ISCOOL output is optimised, the transport of the ion beam through the entire CRIS setup requires tuning. Hence a significant percentage of beam time is spent on beam tuning at the start of an on-line campaign. An independent RFQCB output would be fully optimised before the experiment begins saving valuable time. The atom-laser overlap would also be fully optimised in advance, which is a multidimensional problem and can take many hours to find the best solution to as several lasers are used. A dedicated RFQCB would enable the completion of these tasks offline, in advance of a radioactive (online) campaign. Integration of a new RFQCB with the CRIS ablation ion source would significantly reduce the time associated with switching between stable, and radioactive isotopes of the same element, and enable simultaneous scanning of both masses in some cases. The measurement of isotope shifts and hyperfine structure requires multiple reference scans of stable isotopes. Since the beam from both ISOLDE and the CRIS ablation ion source will pass through the same cooler buncher, the phase space properties for both cases will be reset following the non-conservative, kinematic interactions with the buffer gas. This enables valid reference measurements between stable and radioactive beams originating from different sources. 
(a)

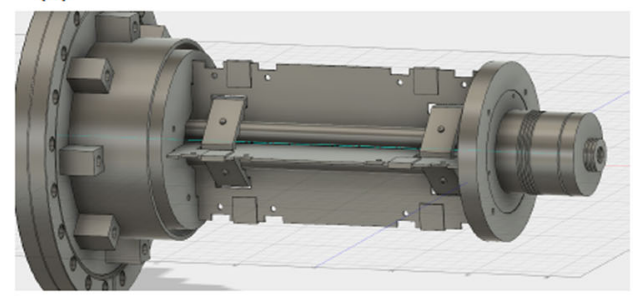

(b)

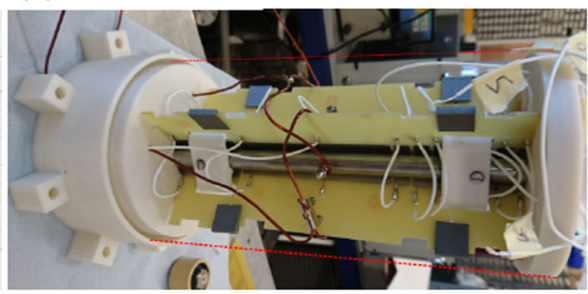

(c)

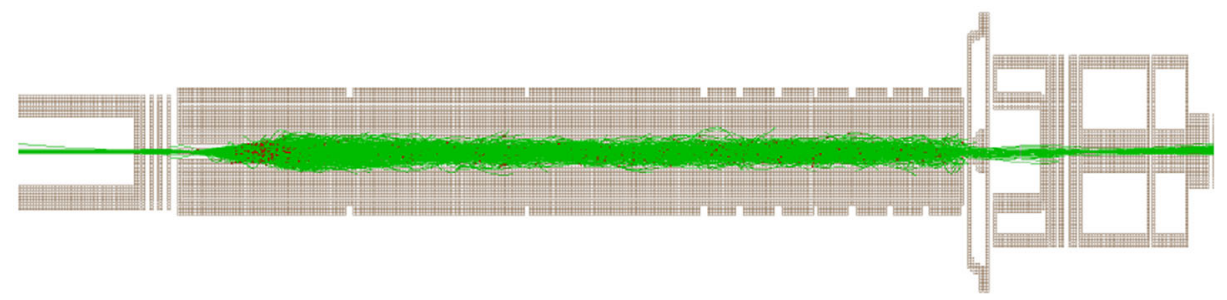

Fig. 1 a CAD rendering of the first prototype. The injection and extraction endcaps are made using a 3D printer. b The assembled prototype, the dotted lines indicate the outer position of a metal jacket which will be filled with a buffer gas. c Ion trajectory simulations using the prototype geometry

\section{The trap prototype}

The RFQCB prototype consists of four longitudinal metal rods positioned in a cross pattern around the beam axis. Separate DC planar electrodes are used to mitigate alignment issues that arise when using a segmented rod design. The design philosophy follows that of ISCOOL [7], except in this case the planar electrodes are copper pads which sit on printed circuit boards (PCBs) and run along the length of the trapping region. These electrodes are independently biased to achieve the desired drag field. Once the optimal DC potential structure is known, later versions of the device may incorporate potential dividers to simplify the application of voltage and reduce the number of power supplies required.

A computer aided design (CAD) rendering of the first prototype is shown in Fig. 1a. This device is housed in a metal jacket with a gas inlet fitted to the side. Two $2 \mathrm{~mm}$ diameter apertures, one at either end of the device provide conductance limitation which aids differential pumping.

A simulation for a short $(20 \mathrm{~cm})$ trap following the geometry of the device prototype shown in Fig. 1a is being developed with the SIMION ion optics package. Initial simulations suggest that a significant number of ions can be trapped over a decreased length, and that the dominant loss mechanism is due to the beam envelope undergoing rapid expansion when injecting and decelerating ions from $\mathrm{keV}$ energies. With an idealised beam the prototype design has close to design specifications in terms of transport efficiency. Ion trajectories are shown in Fig. 1c. These losses may be reduced with alternative injection geometries. A Monte Carlo hard sphere model suggests that $\approx 0.1$ mbar represents the upper limit of operation as significant viscous drag occurs to achieve adequate cooling. Basic flow rate calculations suggest that the regions before and after the trap can be maintained at pressures $<10^{-5}$ mbar for this trap pressure using two stages of differential pumping. A compact prototype has been developed at the University of Manchester and is currently undergoing development and characterisation. The prototype shown in Fig. 1b has a $20 \mathrm{~cm}$ trapping 
Table 1 A selection of the desired characteristics of the RFQCB for CRIS experiments. These values are based of the average performance characteristics of ISCOOL

\begin{tabular}{ll}
\hline Trapping length & $<800 \mathrm{~mm}$ \\
Transmission efficiency & $>50 \%$ \\
Energy spread & $<0.3 \mathrm{eV}$ \\
External pressure & $<5 \times 10^{-6}$ mbar \\
Bunch width & $<3 \mu \mathrm{s}$ \\
\hline
\end{tabular}

length, which is significantly more compact than existing devices [7]. Ongoing design and simulations will look carefully at the potential gradient upon injection, and extraction, the operating pressure, and the vacuum conductance.

RF AC sine, or square wave voltages will be applied to the rods with a 180 degrees phase difference applied to adjacent rods to induce an oscillating field for radial confinement. A DC drag potential is also applied in the trapping region for axial confinement using a system of electrodes separate to the rods. Alternatively, the DC drag field can be achieved with segmentation of the rods and resistively coupling each element in turn [10,11], or by using a graduated cut on the rods coupled with an RF backbone [12]. The device will be held at a high voltage relative to ground in order to decelerate the incoming ion beam from $30 \mathrm{keV}$ to $\approx 100 \mathrm{eV}$. Spatial limitations in the ISOLDE hall require that the new RFQCB is compact. The available space for the whole system which includes the RFQCB, and the injection, extraction, and transport optics is $\approx 2 \mathrm{~m}$. There is therefore a possibility that the device will have to sit off axis, to the side of the beam or be placed in an elevated position. An aim for this project is to house the ablation ion source, and the new RFQCB in one chamber. This would reduce the required space and simplify the application of a float voltage to both elements with the chamber acting as a Faraday cage. This will also enable precision mounting of ion optical elements. A field emission $\mathrm{Ga}$ ion source has been set up for the purpose of RFQCB development. This provides a versatile beam with tunable emittance making it ideal for testing an RFQCB. For a new cooler buncher to be compatible with, and beneficial to the CRIS experiment at ISOLDE, key characteristics must be met, and a list of these are given in Table 1.

\subsection{D printed components}

The compact prototype incorporates many 3D printed and PCB based DC optics and mounting pieces, allowing for rapid prototyping. The printed parts are comprised of a polylactic acid (PLA), a biopolymer derived from plants which is not yet well understood in terms of its dielectric properties and vacuum compatibility. Tests were performed using high performance PLA obtained from Verbatim technologies. An investigation into the UHV compatibility of this material was undertaken. A simple $200 \mathrm{~mm}$ tee chamber was assembled with two $50 \mathrm{~L} / \mathrm{s}$ turbo pumps mounted on orthogonal ports. The turbo pumps were backed by two independent two-stage rotary pumps. Pump-down curves were obtained with and without a $20 \mathrm{~mm}^{3}$ sample of PLA present in the chamber. The test was repeated with samples printed at 100\% (solid) and 30\% (70\% free space) print densities and all data was verified as reproducible. The curves are shown in Fig. 2.

Whilst the PLA outgasses at an elevated rate, the required time before the vacuum pressure reaches levels where device testing can be performed is not excessive, and after several days of pumping the system reached pressures in the $10^{-9}$ mbar range. It was therefore concluded that the PLA material is compatible for applications at pressures above $10^{-8} \mathrm{mbar}$. 


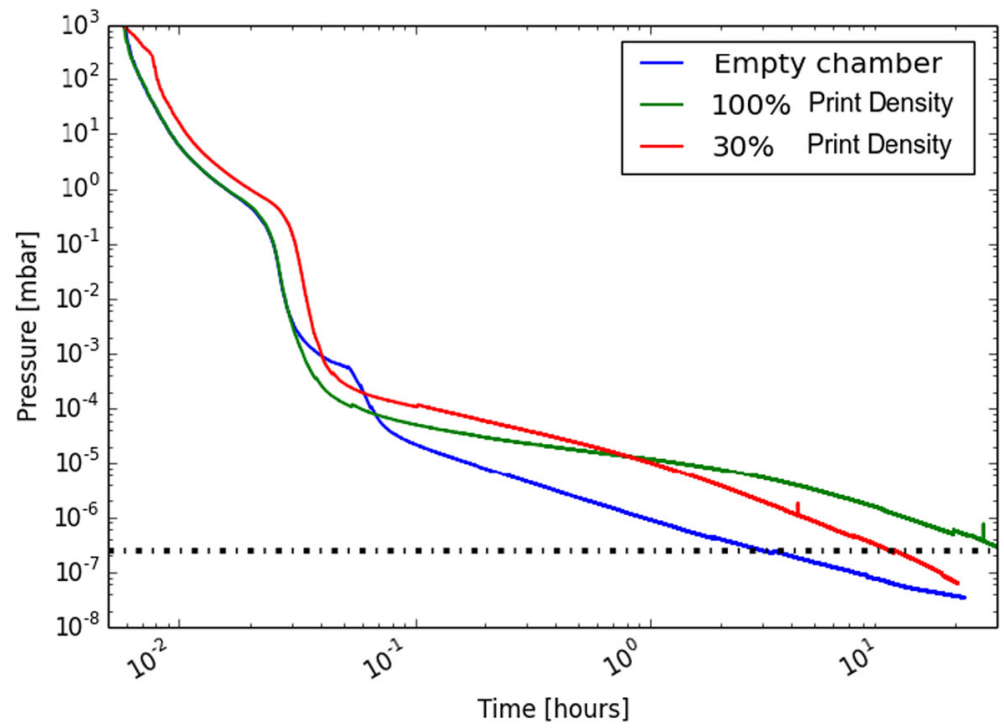

Fig. 2 Pressure as a function of time for the cases of an empty chamber (blue), and PLA samples of 100\% (green), and $30 \%$ (red) print density. The data is plotted on a log scale for clarity. The dashed line indicates a target pressure for in vacuum testing $\left(2.5 \times 10^{-7} \mathrm{mbar}\right)$. This pressure is reached in around 3 hours with an empty chamber, and around 10.5 and 32 hours with the $30 \%$ and $100 \%$ print densities respectively. Early features up to and including the $\approx 10^{-1}$ hour mark are related to pumps switching on, and differences here are not considered to be influenced by the presence of PLA

This enables new designs to be rapidly prototyped and tested. Whilst it takes a few hours to out-gas, a considerable amount of time is saved on workshop and machining processes which can take anything from days to months depending on the complexity of the part to be made, and the workload of the technicians involved. With this system, the printed parts required to fully assemble a prototype RFQCB can be produced in around a day when printing at $100 \%$. We find that a lower print density (30\%) PLA has a higher outgassing rate, likely due to an increased porosity and therefore more efficient desorption. This decreases the pump down time, and drastically reduces the time required to print the component. We find no issues related to the tensile strength of the components printed at this lower density for our applications. The final design will have all larger printed parts remade from UHV compatible metals, ceramics, and PEEK when the prototyping phase is complete.

\subsection{LMIS ion source}

A liquid metal ion source (LMIS) loaded with gallium is currently used to test the RFQCB prototype efficacy. This ion source produces a high current with a beam energy up to 25 $\mathrm{keV}$. Two adjustable irises are used to collimate the beam to a spot size between 1 and 4 $\mathrm{mm}$ in diameter, and the beam has been profiled using a retractable knife edge and Faraday cup. The beam is produced via a field emission process. Liquid gallium is held in a reservoir below a fine tip which is approximately $1 \mu \mathrm{m}$ thick. The potential applied induces a strong electric field which causes the liquid to form an electrospray Taylor-Gilbert cone [13] and ions are produced by field evaporation at the tip of the cone where the electric field is strong. The setup is shown in Fig. 3. 

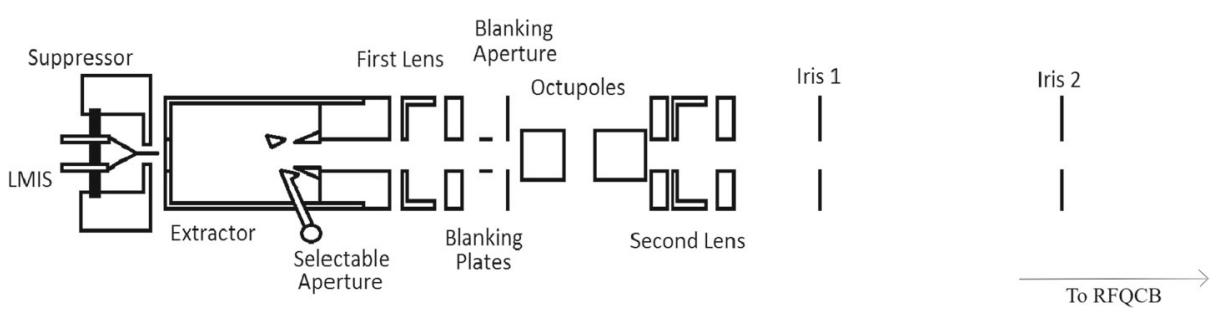

Fig. 3 Layout of the liquid metal ion source. The beam can be collimated using the adjustable irises. This source provides a suitable beam for the initial testing phase of the RFQCB prototype

This versatile ion source facilitates the testing of the prototype. The RFQ parameters will therefore be tuned to cool and bunch stable isotopes of gallium. The device will also be tested with light elements

\section{Conclusions and outlook}

The design and prototyping phase of a new, and dedicated RFQCB for CRIS experiments is in progress. This project will now be focused on the application of both RF and DC voltages, and beam transport and optimisation using a versatile LMIS loaded with gallium. The natural gallium will provide a useful test for trapping its two stable isotopes which occur at an approximate ratio of 60:40 between masses 69 and 71 respectively. Vacuum conductance calculations are also undergoing refinement. Ion trajectory modelling is also being developed in the SIMION package. Considerations will be made for injection and extraction electrode geometries as losses due to beam envelope expansion will need to be minimised following deceleration. The operating pressure for the reduced trapping length will also be integrated into the simulation using a hard sphere collisional model. We have shown that $3 \mathrm{D}$ printed parts made from high quality PLA are vacuum compatible and ideal for testing and development. Lower print densities appear to be more suitable due to a higher initial out gassing rate. However, lower print densities have a reduced tensile strength but a $30 \%$ print density appears to be suitable for these and similar applications. Further tests will be conducted on PLA at the University of Manchester including tests on an increased range of print densities, and alternative sample preparation protocols. Considerations are also being made for an alternative, two-stage design which may have improved vacuum conditions on extraction, and an decreased energy spread due to longer cooling times.

Acknowledgements This work was supported by ERC Consolidator Grant No. 648381 (FNPMLS); STFC Grants No. ST/L005794/1, No. ST/L005786/1 and No. ST/S002316/1; GOA 15/010 from KU Leuven; the FWO-Vlaanderen (Belgium) and the European Union's Horizon 2020 research and innovation programme under Grant Agreement No. 654002 (ENSAR 2). We acknowl- edge the financial aid from the Ed Schneiderman Fund at New York University. B. K. S. acknowledges financial support from Chinese Academy of Science through the PIFI fellowship under Project No. 2017VMB0023 and partly by the TDP project of Physical Research Laboratory (PRL), Ahmedabad. We would like to thank Andrew Mcfarlane for his technical assistance.

Open Access This article is distributed under the terms of the Creative Commons Attribution 4.0 International License (http://creativecommons.org/licenses/by/4.0/), which permits unrestricted use, distribution, and reproduction in any medium, provided you give appropriate credit to the original author(s) and the source, provide a link to the Creative Commons license, and indicate if changes were made. 


\section{References}

1. Cocolios, T.E., Al Suradi, H.H., Billowes, J., Budinevi, I., de Groote, R.P., De Schepper S., Fedosseev, V.N., Flanagan, K.T., Franchoo, S., Garcia Ruiz, R.F., Heylen, H., Le Blanc, F., Lynch, K.M., Marsh, B.A., Mason, P.J.R., Neyens, G., Papuga, J., Procter, T.J., Rajabali, M.M., Rossel, R.E., Rothe, S., Simpson, G.S., Smith, A.J., Strashnov, I., Stroke. H. H., Verney, D., Walker, P.M., Wendt, K.D.A., Wood, R.T.: The Collinear Resonance Ionization Spectroscopy (CRIS) experimental setup at CERN-ISOLDE. Nuclear Instruments and Methods in Physics Research Section B: Beam Interactions with Materials and Atoms",XVIth International Conference on ElectroMagnetic Isotope Separators and Techniques Related to their Applications, December 2-7, 2012 at Matsue, Japan

2. de Groote, R.P., Billowes, J., Binnersley, C.L., Bissell, M.L., Cocolios, T.E., Day Goodacre, T., FarooqSmith, G.J., Fedorov, D.V., Flanagan, K.T., Franchoo, S., Garcia Ruiz, R.F., Koszorús, Á., Lynch, K.M., Neyens, G., Nowacki, F., Otsuka, T., Rothe, S., Stroke, H.H., Tsunoda, Y., Vernon, A.R., Wendt, K.D.A., Wilkins, S.G., Xu, Z.Y., Yang, X.F.: Dipole and quadrupole moments of ${ }^{73-78} \mathrm{Cu}$ as a test of the robustness of the $Z=28$ shell closure near ${ }^{78} \mathrm{Ni}$

3. Farooq-Smith, G.J., Vernon, A.R., Billowes, J., Binnersley, C.L., Bissell, M.L., Cocolios, T.E., Day Goodacre, T., de Groote, R.P., Flanagan, K.T., Franchoo, S., Garcia Ruiz, R.F., Gins, W., Lynch, K.M., Marsh, B.A., Neyens, G., Rothe, S., Stroke, H.H., Wilkins, S.G., Yang, X.F.: Probing the ${ }_{31}$ Ga groundstate properties in the region near $Z=28$ with highresolution laser spectroscopy. Phys. Rev. C 96(4), 044324 (2017). https://doi.org/10.1103/PhysRevC.96.044324

4. Lynch, K.M., Wilkins, S.G., Billowes, J., Binnersley, C.L., Bissell, M.L., Chrysalidis, K., Cocolios, T.E., Goodacre, T.D., de Groote, R.P., Farooq-Smith, G.J., Fedorov, D.V., Fedosseev, V.N., Flanagan, K.T., Franchoo, S., Garcia Ruiz, R.F., Gins, W., Heinke, R., Koszorús, Á., Marsh, B.A., Molkanov, P.L., Naubereit, P., Neyens, G., Ricketts, C.M., Rothe, S., Seiffert, C., Seliverstov, M.D., Stroke, H.H., Studer, D., Vernon, A.R., Wendt, K.D.A., Yang, X.F.: Laser-spectroscopy studies of the nuclear structure of neutron-rich radium. Phys. Rev. C 97(2), 024309 (2018). https://doi.org/10.1103/PhysRevC.97.024309

5. Wilkins, S.G., Lynch, K.M., Billowes, J., Binnersley, C.L., Bissell, M.L., Cocolios, T.E., Goodacre, T.D., de Groote, R.P., Farooq-Smith, G.J., Flanagan, K.T., Franchoo, S., Ruiz, R.F.G., Gins, W., Heylen, H., Koszorús, Á., Neyens, G., Stroke, H.H., Vernon, A.R., Wendt, K.D.A., Yang, X.F.: Quadrupole moment of ${ }^{203}$ Fr. Phys. Rev. C 96(3), 034317 (2017). https://doi.org/10.1103/PhysRevC.96.034317

6. Catherall, R., Andreazza, W., Breitenfeldt, M., Dorsival, A., Focker, G.J., Gharsa, T.P., Giles, T.J., Grenard, J.L., Locci, F., Martins, P., Marzari, S., Schipper, J., Shornikov, A., Stora, T.: The ISOLDE facility. J. Phys. G: Nucl. Part. Phys. 44(9), 094002 (2017)

7. Mané, E., Billowes, J., Blaum, K., Campbell, P., Cheal, B., Delahaye, P., Flanagan, K., Forest, D., Franberg, H., Geppert, C., Giles, T., Jokinen, A., Kowalska, M., Neugart, R., Neyens, G., Nörtershäuser, W., Podadera, I., Tungate, G., Vingerhoets, P., Yordanov, D.T.: An ion cooler-buncher for high-sensitivity collinear laser spectroscopy at ISOLDE. Eur. Phys. J. A 42, 503-507 (2009). https://doi.org/10.1140/epja/ i2009-10828-0

8. Garcia Ruiz, R.F., Vernon, A.R., Binnersley, C.L., Sahoo, B.K., Bissell, M., Billowes, J., Cocolios, T.E., Gins, W., de Groote, R.P., Flanagan, K.T., Koszorus, A., Lynch, K.M., Neyens, G., Ricketts, C.M., Wendt, K.D.A., Wilkins, S.G., Yang, X.F.: High-precision multiphoton ionization of accelerated laserablated species. Phys. Rev. X 8(4), 041005 (2018)

9. Klose, A., Minamisono, K., Geppert, C.h., Frömmgen, N., Hammen, M., Krämer, J., Krieger, A., Levy, C.D.P., Mantica, P.F., Nörtershäuser, W., Vinnikova, S.: Tests of atomic charge-exchange cells for collinear laser spectroscopy. Nucl. Instrum. Methods Phys. Res., Sect. A: Accelerators, Spectrometers, Detectors and Associated Equipment 678, 114-121 (2012)

10. Nieminen, A., Huikari, J., Jokinen, A., Äystö, J., Campbell, P., Cochrane, E.C.A.: Beam cooler for low-energy radioactive ions. Nucl. Instrum. Methods Phys. Res., Sect. A: Accelerators, Spectrometers, Detectors and Associated Equipment 469(2), 244-253 (2001)

11. Smith, M.: A square-wave-driven radiofrequency quadrupole cooler and buncher for titan (2005)

12. Minamisono, K., Mantica, P.F., Klose, A., Vinnikova, S., Schneider, A., Johnson, B., Barquest, B.R.: Commissioning of the collinear laser spectroscopy system in the BECOLA facility at NSCL. Nucl. Instrum. Methods Phys. Res., Sect. A: Accelerators, Spectrometers, Detectors and Associated Equipment 709, 85-94 (2013). ISSN 0168-9002

13. Fenn, J.B., Mann, M., Meng, C.K., Wong, S.F., Whitehouse, C.M.: Electrospray ionization for mass spectrometry of large biomolecules. Science 246(4926), 64-71 (1989)

Publisher's note Springer Nature remains neutral with regard to jurisdictional claims in published maps and institutional affiliations. 